\title{
Acute kidney injury after cardiac and non-cardiac surgery: are there more similarities than differences?
}

\author{
Mark Stafford-Smith
}

Received: 30 March 2015/Accepted: 13 April 2015/Published online: 23 April 2015

(c) Canadian Anesthesiologists' Society 2015

All acute nephropathies are not created equal. Radiocontrast dye, various pigments (e.g., myoglobin, hemoglobin), thrombi, uric acid, nephrotoxic medications (e.g., aminoglycosides for the treatment of sepsis), as well as cholesterol and atheroemboli are all sources of acute kidney injury (AKI) seen in the perioperative period. ${ }^{1}$ Furthermore, while each AKI subtype is associated with considerable morbidity and mortality, their characteristic setting, risk factors, and therapies vary widely. In some cases, AKI preventive therapy can be quite effective, e.g., early forced alkaline diuresis to alleviate pigment nephropathy; ${ }^{2}$ however, for most AKI types, "therapeutic" strategies are limited to measures aimed at reducing further exposure to any ongoing source of insult (e.g., sepsis).

One of the most studied, though arguably least understood, AKI conditions is that which occurs following cardiac surgery - so-called cardiac surgery-associated AKI (CSA-AKI). ${ }^{3}$ Since this syndrome was first recognized almost fifty years ago, it has been meticulously described relative to its relationship with various risk factors and to variation in clinical practice; however, much is still not known regarding its underlying pathophysiology. To date, there is no effective treatment once CSA-AKI has occurred, and identifiable predictors explain only $10-15 \%$ of the variability in the likelihood of its occurrence. ${ }^{4}$

Although evidence points towards CSA-AKI being a multifactorial condition involving numerous sources of perioperative renal insult, cardiopulmonary bypass (CPB) and its unique impact on renal physiology has been incriminated as a central factor. ${ }^{5,6}$ For example, in

M. Stafford-Smith $(\bowtie)$

Department of Anesthesiology, Duke University Medical Center,

Box 3094 DUMC, Durham, NC 27710, USA

e-mail: mark.staffordsmit@dm.duke.edu retrospective studies, the duration of $\mathrm{CPB}$ consistently predicts an increased likelihood of AKI. ${ }^{7}$ Nevertheless, the story is not so simple, as extended periods of CPB usually imply more complex surgery which itself can impact the risk of AKI. In addition, primary elective coronary artery bypass surgery with CPB is typically relatively unaffected by significant AKI. Furthermore, equivalent cardiac surgery procedures performed either off or on bypass can be difficult to differentiate by AKI risk. ${ }^{8}$ Nonetheless, cardiac surgery is so often the source of perioperative dialysis that, for a long while, it was considered the only cause of serious postoperative AKI.

Operations not involving the heart (i.e., non-cardiac surgery) include a heterogeneous - by location and invasiveness - collection of procedures that often avoid the scrutiny and daily determination of serum creatinine in the intensive care unit (ICU). Hence, the spectrum of AKI complicating the approximately 200 million non-CSA procedures that occur worldwide annually is relatively unexplored. ${ }^{9}$ Nevertheless, in cohorts of non-CSA patients admitted to ICUs, AKI turns out to be much more common than previously thought. In one study, Abelha et al. noted an AKI rate of $7.5 \%$ in 1,166 patients with normal preoperative renal function. ${ }^{10}$ These authors also found that AKI had a risk factor profile which resembled that seen in cardiac patients with the onset of AKI and predicted similarly poor outcomes.

While the existence of non-CSA-AKI as an important condition is now acknowledged, this condition remains far less understood than CSA-AKI. Major procedures known to be commonly complicated by AKI now include major vascular surgery, liver transplants, esophagogastrectomy, gastric bypass, thoracotomy, liver resections, and total knee replacements. ${ }^{11,12}$ As highlighted above, different AKI syndromes occur in varied settings and with a spectrum of risk factors (e.g., rhabdomyolysis) that sometimes advocate for specific therapies. 
Consequently, non-CSA-AKI must be systematically compared and contrasted with CSA-AKI. Non-CSA-AKI itself may represent multiple conditions that relate to the different types of surgeries. Clearly, insults unique to cardiac surgery, such as $\mathrm{CPB}$, intra-aortic balloon counterpulsation, and circulatory arrest, are not pertinent to non-cardiac surgery settings, but much remains to be understood about this condition.

Two separate retrospective studies in the current edition of the Journal extend our understanding of perioperative renal function and AKI in the setting of non-cardiac surgery. Cywinski et al. have highlighted the importance of using the most precise tools available to estimate renal filtration function as a perioperative variable. In their analysis of more than 90,000 patients, the authors compared serum creatinine alone with an estimate of glomerular filtration rate, in this case to determine the best prediction of mortality risk. ${ }^{13}$ In a second study, Tomozawa et al. found preoperative hypertension, intraoperative transfusion, and pre-existing renal dysfunction to be AKI risk factors in a cohort of 642 patients undergoing major liver resection. ${ }^{14}$ This second study also links AKI with increased hospital length of stay, risk of respiratory failure, and in-hospital mortality. These observations align with those made with other non-cardiac surgery cohorts as well as with cardiac surgery populations.

As more is understood about perioperative AKI, following both cardiac and non-cardiac procedures, advances in other AKI research represent a coordinated strategy to prepare the way for identifying better approaches for the prevention and treatment of both types of AKI. Such methods include the identification of better early biomarkers to facilitate prompt intervention and even novel preventive therapies that appear to have promise in both CSA and nonCSA settings (e.g., remote ischemic preconditioning). ${ }^{15-17}$ The observations of risk factors and consequences of AKI in non-CSA patients reported in this month's issue of the Journal add to our growing collective knowledge of likely important overlap in the risk factors for and consequences of AKI between CSA and non-CSA procedures.

\section{L'insuffisance rénale aiguë après une chirurgie cardiaque ou non cardiaque: plus de similitudes que de différences?}

Toutes les néphropathies aiguës ne se valent pas. La teinture de contraste radiologique, divers pigments (par ex., la myoglobine, l'hémoglobine), les thrombus, l'acide urique, les médicaments néphrotoxiques (par ex., les aminosides utilisés pour le traitement d'une sepsie), ainsi que le cholestérol et les athéroembolies sont tous des sources d'insuffisance rénale aiguë (IRA) qu'on peut observer en période périopératoire. ${ }^{1}$ De plus, bien que chaque sous-type d'IRA soit associé à une morbidité et une mortalité importantes, les spécificités de leur contexte, de leurs facteurs de risque et de leur traitement varient grandement. Dans certains cas, le traitement préventif de l'IRA peut être assez efficace. Par exemple, on peut utiliser une diurèse alcaline forcée précoce pour soulager une néphropathie due aux pigments. ${ }^{2}$ Toutefois, dans la plupart des types d'IRA, les stratégies «thérapeutiques » se limitent à des mesures dont l'objectif est de réduire toute exposition supplémentaire à toute source continue d'agression (par ex., une sepsie).

L'une des conditions d'IRA les plus étudiées, tout en étant probablement l'une des moins bien comprises, est l'IRA qui survient après une chirurgie cardiaque. ${ }^{3}$ Depuis la première identification de ce syndrome il $\mathrm{y}$ a près de cinquante ans, sa relation à divers facteurs de risque et aux variations de la pratique clinique a été décrite de façon très détaillée. En revanche, nous ne connaissons que peu de choses quant à sa physiopathologie sous-jacente. À ce jour, il n'existe pas de traitement efficace une fois qu'une IRA associée à la chirurgie cardiaque se manifeste, et les prédicteurs identifiables n'expliquent que $10-15 \%$ de la variabilité de sa probabilité de survenue. ${ }^{4}$

Bien que les données probantes dont nous disposons laissent à penser que l'IRA associée à la chirurgie cardiaque soit une maladie multifactorielle liée à plusieurs sources d'agression rénale périopératoire, la circulation extracorporelle (CEC) et son impact singulier sur la physiologie rénale ont été incriminés comme le facteur principal. ${ }^{5,6}$ Par exemple, dans les études rétrospectives, la durée de la CEC est un prédicteur constant d'une probabilité accrue d'IRA. ${ }^{7}$ L'explication n'est pas si simple cependant: en effet, des périodes prolongées de CEC impliquent en général des chirurgies plus complexes, ce qui pourrait également avoir un impact sur le risque d'IRA. De plus, les chirurgies non urgentes de première intention de pontages coronariens avec une $\mathrm{CEC}$ ne sont en règle générale pas affectées par une importante IRA. En outre, des interventions de chirurgie cardiaque équivalentes, réalisées sous CEC ou non, peuvent être difficiles à différencier en fonction du risque associé d'IRA. ${ }^{8}$ Ceci étant dit, la chirurgie cardiaque est si souvent la source de dialyse périopératoire qu'elle a pendant longtemps été considérée comme la cause unique d'IRA postopératoire grave.

Les opérations qui ne touchent pas au cœur (c.-à-d. les chirurgies non cardiaques) forment une collection hétérogène - de par leur localisation et leur degré 
« d'invasivité » - d'interventions qui passent bien souvent à côté de l'examen minutieux et de la détermination quotidienne de la créatininémie à l'unité des soins intensifs (USI). Pour cette raison, l'ampleur des IRA compliquant les quelques 200 millions d'interventions hors chirurgie cardiaque qui surviennent chaque année dans le monde est relativement peu connue. ${ }^{9}$ Toutefois, dans les cohortes de patients hors chirurgie cardiaque admis dans les USI, les IRA sont bien plus courantes qu'on ne l'imaginait par le passé. Dans une étude, Abelha et coll. ont noté un taux d'IRA de 7,5\% chez 1166 patients ayant une fonction rénale préopératoire normale. ${ }^{10}$ Ces auteurs ont également observé que l'IRA avait un profil de facteurs de risque proche de celui de patients cardiaques avec l'apparition d'une IRA et laissait présager des pronostics tout aussi sombres.

Bien que l'existence de l'IRA non associée à la chirurgie cardiaque en tant que maladie grave soit désormais reconnue, cette condition demeure beaucoup moins bien comprise que son pendant associé à la chirurgie cardiaque. Les interventions majeures connues comme étant souvent compliquées par une IRA incluent dorénavant les chirurgies vasculaires majeures, les greffes hépatiques, l'oesophagogastrectomie, le pontage gastrique, la thoracotomie, les résections hépatiques et les arthroplasties totales du genou. ${ }^{11,12}$ Comme nous l'avons souligné ci-dessus, différents syndromes d'IRA surviennent dans différents contextes et sont associés à un éventail de facteurs de risque (par ex., la rhabdomyolyse) qui préconisent des traitements spécifiques.

C'est pourquoi les IRA hors chirurgie cardiaque doivent être systématiquement comparées aux et contrastées avec les IRA associées à la chirurgie cardiaque. L'IRA hors chirurgie cardiaque peut elle-même être constituée de nombreuses maladies liées aux différents types de chirurgie. Clairement, les agressions spécifiques à la chirurgie cardiaque, telles que la CEC, les ballons de contre-pulsion intra-aortique et l'arrêt circulatoire, ne s'appliquent pas aux contextes hors chirurgie cardiaque, mais nous avons encore beaucoup de choses à comprendre concernant cette maladie.

Deux études rétrospectives distinctes publiées dans ce numéro du Journal approfondissent notre compréhension de la fonction rénale périopératoire et de l'IRA dans un contexte de chirurgie non cardiaque. Cywinski et coll. ont souligné l'importance d'utiliser les outils les plus précis qui soient afin d'estimer la fonction de filtration rénale en tant que variable périopératoire. Dans leur analyse, qui porte sur plus de 90000 patients, les auteurs ont comparé la créatininémie seule à un estimé de débit de filtration glomérulaire, dans ce cas afin de déterminer le meilleur prédicteur du risque de mortalité. ${ }^{13}$ Dans une autre étude, Tomozawa et coll. ont observé que l'hypertension préopératoire, la transfusion peropératoire et une dysfonction rénale préexistante constituaient des facteurs de risque d'IRA dans une cohorte de 642 patients subissant une résection hépatique majeure. ${ }^{14}$ Cette deuxième étude associe également l'IRA à une durée d'hospitalisation prolongée, à un risque d'insuffisance respiratoire et à une mortalité hospitalière accrue. Ces observations font écho à celles d'autres cohortes hors chirurgie cardiaque ainsi que de populations de patients de chirurgie cardiaque.

Au fur et à mesure que notre compréhension de l'IRA périopératoire s'améliore, qu'elle survienne après une intervention de chirurgie cardiaque ou non cardiaque, tous les progrès concernant les autres IRA formeront une stratégie coordonnée qui permettra de paver la voie pour la découverte d'approches plus efficaces de prévention et de traitement de ces deux types d'IRA. De telles méthodes comprennent l'identification de meilleurs biomarqueurs précoces afin de faciliter une intervention rapide, ou encore certaines thérapies préventives innovantes qui semblent prometteuses tant dans le cadre d'une chirurgie cardiaque que non cardiaque (par ex., le préconditionnement ischémique à distance). ${ }^{15-17}$ Les observations concernant les facteurs de risque et les conséquences de l'IRA chez les patients hors chirurgie cardiaque rapportées dans ce numéro du Journal s'ajoutent à nos connaissances collectives concernant le chevauchement probablement important des facteurs de risque et des conséquences de l'IRA entre les interventions cardiaques et non cardiaques.

Conflicts of interest None declared.

Conflit d'intérêt Aucun.

\section{References}

1. Greenberg A. Acute kidney injury (book section). In: Greenberg A (Ed.). National Kidney Foundation's Primer on Kidney Diseases, 5th ed. Philadelphia, PA: Saunders Elsevier; 2009: 269-312.

2. Nath AK, Murali NS. Myoglobinuric and hemoglobinuric acute kidney injury. In: Greenberg A (Ed.). National Kidney Foundation's Primer on Kidney Diseases, 5th ed. Philadelphia, PA: Saunders Elsevier; 2009.

3. Thiele RH, Isbell JM, Rosner MH. AKI associated with cardiac surgery. Clin J Am Soc Nephrol 2015; 10: 500-14.

4. Stafford-Smith $M$, Podgoreanu $M$, Swaminathan $M$, et al. Association of genetic polymorphisms with risk of renal injury after coronary bypass graft surgery. Am J Kidney Dis 2005; 45: 519-30.

5. Stafford-Smith M, Grocott HP. Renal medullary hypoxia during experimental cardiopulmonary bypass: a pilot study. Perfusion 2005; 20: 53-8.

6. Garg AX, Devereaux PJ, Yusuf S, et al. Kidney function after offpump or on-pump coronary artery bypass graft surgery: a randomized clinical trial. JAMA 2014; 311: 2191-8.

7. Conlon PJ, Stafford-Smith M, White WD, et al. Acute renal failure following cardiac surgery. Nephrol Dial Transplant 1999; 14: $1158-62$. 
8. Gamoso MG, Phillips-Bute B, Landolfo KP, Newman $M F$, Stafford-Smith $M$. Off-pump versus on-pump coronary artery bypass surgery and postoperative renal dysfunction. Anesth Analg 2000; 91: 1080-4.

9. Weiser TG, Regenbogen SE, Thompson KD, et al. An estimation of the global volume of surgery: a modelling strategy based on available data. Lancet 2008; 372: 139-44.

10. Abelha FJ, Botelho M, Fernandes V, Barros $H$. Determinants of postoperative acute kidney injury. Crit Care 2009; 13: R79.

11. Josephs SA, Thakar CV. Perioperative risk assessment, prevention, and treatment of acute kidney injury. Int Anesthesiol Clin 2009; 47: 89-105.

12. Sehgal V, Bajwa SJ, Sehgal R, Eagan J, Reddy P, Lesko SM. Predictors of acute kidney injury in geriatric patients undergoing total knee replacement surgery. Int J Endocrinol Metab 2014; 12: e16713.

13. Cywinski JB, Mascha EJ, Kurz A, Sessler DI. Estimated glomerular filtration rate better predicts 30-day mortality after non-cardiac surgery than serum creatinine: a retrospective analysis of 92,888 patients. Can J Anesth 2015; 62; DOI:10. 1007/s12630-015-0398-8.

14. Tomozawa A, Ishikawa S, Shiota N, Cholvisudhi P, Makita K. Perioperative risk factors for acute kidney injury after liver resection surgery: an historical cohort study. Can J Anesth 2015; 62; DOI: 10.1007/s12630-015-0397-9.

15. Haji Mohd Yasin NA, Herbison P, Saxena P, Praporski S, Konstantinov IE. The role of remote ischemic preconditioning in organ protection after cardiac surgery: a meta-analysis. J Surg Res 2014; 186: 207-16.

16. Candilio L, Malik A, Ariti C, et al. Effect of remote ischaemic preconditioning on clinical outcomes in patients undergoing cardiac bypass surgery: a randomised controlled clinical trial. Heart 2015; 101: 185-92.

17. Li C, Хи M, Wu Y, Li YS, Huang WQ, Liu KX. Limb remote ischemic preconditioning attenuates lung injury after pulmonary resection under propofol-remifentanil anesthesia: a randomized controlled study. Anesthesiology 2014; 121: 249-59. 